\title{
Frequent occurrence of undiagnosed pelvic inflammatory disease in remote communities of central Australia
}

Bronwyn J Silver

BNurs, Gra

Research Fellow

Janet Knox

MMed(STD/HIV)

DTM\&H, FAChSHM

Sexual Health Physician

Kirsty S Smith

GDipCommNurs,

GDipEd, MPH,

Sexual Health

Nurse Consultant ${ }^{2}$

James S Ward

Deputy Director

Jacqueline Boyle

FRANZCOG, MPH, PhD,

Obstetrician and

Gynaecologist and

Senior Research Fellow

Rebecca J Guy

BAppSc, MAppEpid, PhD

Post Doctoral

Research Fellow

John Kaldor PhD,

Professor of Epidemiology and Head of Public Health

Interventions Research

Program²

Alice R Rumbold

BSc(Hons), MPH, PhD Senior Research Fellow 1,5

1 Epidemiology and Health

Systems, Menzies School

of Health Research,

Alice Springs, NT.

2 The Kirby Institute for Infection and

Immunity in Society, University

of New South Wales,

Sydney, NSW.

3 Preventative Health

Baker IDI Heart and

Diabetes Institute

Alice Springs, NT.

4 Jean Hailes Foundation

for Women's Health,

Monash University,

Melbourne, VIC

5 Obstetrics and Gynaecology, University of Adelaide

Adelaide, SA

bronwyn.silver@ menzies.edu.au

MJA 2012; 197: 647-651 doi:10.5694/mjall.11450

Editorial p 597 Research p 642 elvic inflammatory disease (PID) is associated with severe reproductive sequelae, including ectopic pregnancy and tubal factor infertility. ${ }^{1,2}$ It has an infectious origin, and chlamydia and gonorrhoea are important causes of PID in populations where sexually transmissible infections (STIs) occur at high levels. ${ }^{1,3}$

Early detection and effective management of PID in primary care settings can prevent complications and hospitalisation. ${ }^{4}$ Under Australian and international clinical guidelines, ${ }^{5-7}$ clinicians are encouraged to have a high index of suspicion and a low threshold for treatment when a young woman presents with lower abdominal pain, after exclusion of other causes. Treatment includes a 2 -week broad-spectrum antibiotic regimen to cover the likely causative pathogens.

In the Northern Territory, hospitalisation for acute PID occurs at nine times the rate among Aboriginal and Torres Strait Islander women, compared with non-Indigenous women, ${ }^{8}$ and many remote communities have been found to have an increased prevalence of bacterial STIs. ${ }^{9}$ These findings suggest that Aboriginal women may not be receiving effective primary health care for diagnosis and treatment of PID. To assess the extent of PID and its management in remote primary health care centres, we undertook a retrospective study of women presenting with lower abdominal pain, which is the most common symptom of PID.

\section{Methods}

The study was conducted in five primary health care centres in remote central Australia, where high prevalences of gonorrhoea and chlamydia have been recorded in communitywide STI screening programs. ${ }^{9}$ Participating services included government and Aboriginal communitycontrolled facilities. Health care in

Objective: To assess the extent of diagnosed and undiagnosed pelvic inflammatory disease (PID) in Aboriginal women in remote central Australia.

Design, setting and subjects: Retrospective cross-sectional study in five remote central Australian primary health care centres. Medical records of all resident Aboriginal women aged 14-34 years were examined. Data were from presentations with documented lower abdominal pain, excluding other causes, for 2007-2008.

Main outcome measures: PID investigations undertaken, PID diagnoses made, recommended treatment, and presentations meeting the guideline criteria for diagnosing PID based on pelvic examination, symptom profile or history.

Results: Of 655 medical records reviewed, 119 women (18\%) presented 224 times with lower abdominal pain. Recommended investigations to diagnose PID were infrequently undertaken: bimanual examination (15 cases [7\%]); testing for gonorrhoea and chlamydia (78 [35\%]); and history taking for vaginal discharge (59 [26\%]), intermenstrual bleeding (27 [12\%]) and dyspareunia (17 [8\%]). There were 95 presentations (42\%) consistent with guidelines to diagnose PID, most (87 [39\%]) based on symptom profile and history. Of these, practitioners made 15 diagnoses of PID, and none had the recommended treatment documented.

Conclusion: Pelvic inflammatory disease occurred frequently among Aboriginal women in central Australia during the study period but was vastly underdiagnosed and poorly treated. Undiagnosed or inadequately treated PID leads to poorer reproductive health outcomes in the long term. Increased awareness of PID symptoms, diagnosis and treatment and a revision of the guidelines is needed to improve detection and management of PID in this highrisk setting.

these centres is routinely provided by remote area nurses (RANs), with medical advice available by telephone; general practitioners, who attend monthly; and obstetrics and gynaecology health professionals who provide a 6 -monthly clinic. Few centres employ a full-time GP. At the time of the study, these services used a combination of paper-based and electronic systems to record patient information.

We examined all available medical records of resident Aboriginal women aged 14-34 years, to identify all instances of women presenting with lower abdominal pain from 1 January 2007 to 31 December 2008 at participating centres. We excluded women for whom gastroenteritis, trauma, miscarriage or pregnancy (including early labour) were documented as the cause of lower abdominal pain.

For each presentation, we obtained information including:

- the woman's age
- results of gonorrhoea or chlamydia tests the woman had had during 2007 and 2008

- the woman's history of PID

- signs of PID when a bimanual examination was undertaken

- documentation of presence or absence of other signs and symptoms associated with PID (eg, vaginal discharge, dyspareunia and intermenstrual bleeding)

- the diagnosis documented by the practitioner

- the treatment administered

- the attending practitioner type.

In addition to reviewing the medical records at the centres, we obtained information on gonorrhoea and chlamydia testing conducted in the participating centres from a database at the NT Centre for Disease Control, which receives all results of STI testing direct from pathology laboratories.

For each included case of lower abdominal pain, the documented clinical assessments and their out- 
1 Criteria for diagnosis and treatment of pelvic inflammatory disease according to the clinical guidelines for Northern Territory remote health practitioners, 2007-2008

If a bimanual examination is conducted:

- lower abdominal pain and cervical excitation or adnexal tenderness or uterine tenderness

If a bimanual examination is not conducted:

- lower abdominal pain and vaginal discharge; or

- no vaginal discharge and lower abdominal pain, and aged 14-34 years and dyspareunia or intermenstrual bleeding or history of sexually transmitted infection or pelvic inflammatory disease in the past year

comes were assessed against the criteria for diagnosing PID which are specified in guidelines applicable at the time of the study (Box 1). ${ }^{10,11}$ We identified presentations that were documented as having met the criteria for diagnosing PID and, from these, calculated the proportion with a documented diagnosis of PID.

Presentations with documentation that met the guideline criteria, but that had not had a PID diagnosis documented in the medical record, were considered undiagnosed PID. For presentations in which PID was diagnosed, we assessed practitioner adherence to the recommended PID treatment (Box $2)^{10,11}$ and calculated the proportion of presentations with a documented diagnosis of PID for which the correct treatment was prescribed.

Proportions, means and medians were used to summarise the characteristics of presenting women, practitioner types, assessments undertaken, diagnoses made and treatments administered. All analyses were performed using Stata, version 10.1 (StataCorp).

Consultations with the relevant local stakeholders and communities were undertaken before the study. Ethics approval was granted by the Central Australian Human Research Ethics Committee, the Human Research Ethics Committee of the Northern Territory Department of Health and Menzies School of Health controlled health services.

\section{Results}

\section{Characteristics of participating women} (three women).
Research and its associated Aboriginal Ethics Sub Committee, and the University of New South Wales Human Research Ethics Committee. Approval was obtained from the Northern Territory Department of Health and Families and from the boards of the participating Aboriginal community-

Participating health centres held the medical records of 655 eligible women that were available for review. Of these, after the exclusion of other causes of abdominal pain, 119 women (18\%) presented 224 times with lower abdominal pain during the study period 2007-2008 (range, 1-13 presentations per woman; median, two presentations; mean time between presentations, 65 days). The median age was 25.3 years (interquartile range, 20.6-29.6 years). A record of confirmed gonorrhoea and/or chlamydia infection within 90 days before or after the presentation date was found for 62 presentations (28\%) (38 women). A record of any STI in the year before presentation was documented for 78 presentations (35\%) (46 women), and a history of PID was noted in four presentations (2\%)

\footnotetext{
2 Recommended treatment regimen for pelvic inflammatory disease according to the clinical guidelines for Northern Territory remote health practitioners, 2007-2008 ${ }^{10,11}$

Day 1:

- ceftriaxone 250 mg intramuscularly or intravenously (single dose), and

- azithromycin 1 g orally (single dose)

Day 2:

- doxycycline $100 \mathrm{mg}$ orally twice a day for 14 days (doxycycline course can be omitted if azithromycin is administered on day 7), and

- metronidazole 400 mg orally twice a day for 14 days

Day 7:

- azithromycin 1 g orally (single dose)
}

\section{Characteristics of health practitioners}

Of the presentations, $162(72 \%)$ were managed by an RAN, who referred to a GP at 13 presentations (6\%). Care was provided by Aboriginal health workers for 14 presentations (6\%), by GPs for $14(6 \%)$, by sexual or women's health specialists for eight (4\%), and by medical students for two (1\%). For 23 presentations (10\%), the practitioner type was not recorded.

\section{Clinical assessment}

Based on available records, the 224 presentations of 119 women with lower abdominal pain led to a bimanual examination in 15 presentations (7\%) (13 women); and documentation of the presence or absence of vaginal discharge, intermenstrual bleeding and dyspareunia in 59 presentations (26\%) (41 women), 27 presentations (12\%) (22 women) and 17 presentations (8\%) (17 women) respectively (Box 3 ). There were positive findings recorded for about half the bimanual examinations, and less than a quarter of the symptom assessments (Box 3). In addition, testing for gonorrhoea and chlamydia was undertaken in 78 presentations $(35 \%)$ (63 women), with $21 \%$ of gonorrhoea test results positive and $15 \%$ of chlamydia test results positive.

Overall, 35 (16\%) of the 224 presentations with lower abdominal pain had a PID diagnosis recorded. These occurred in 29 women (six women were diagnosed with PID on two occasions). Of these presentations, the attending practitioner type was predominantly an RAN (for 26 presentations). Practitioner type was a GP for three presentations, a women's or sexual health specialist for three presentations and was unrecorded for three presentations. The frequency of investigations undertaken was similar in presentations with a recorded diagnosis of PID and no diagnosis.

\section{Presentations documented as meeting criteria for PID}

Of the 224 presentations (119 women) with lower abdominal pain, we identified 95 presentations (42\%) (54 women) documented as having met the criteria for diagnosing PID according to the guidelines. Of these, 
3 Clinical findings in women aged 14-34 years with lower abdominal pain, 2007-2008 $(n=224)$

Clinical assessment

Undocumented (\%)

Documented (\%)

Bimanual pelvic examination signs

Cervical excitation

$209(93 \%)$

$15(7 \%)[8]$

Adnexal/uterine tenderness

$214(96 \%)$

$10(4 \%)[6]$

Symptoms

Vaginal discharge

$165(74 \%)$

$197(88 \%)$

$59(26 \%)[16]$

Intermenstrual bleeding

$207(92 \%)$

$27(12 \%)[6]$

Dyspareunia eight presentations (seven women) met the criteria based on the detection of cervical excitation and/or adnexal or uterine tenderness on pelvic examination, 11 presentations (seven women) met the criteria based on the presence of vaginal discharge, and 76 presentations (44 women) met the criteria based on a combination of risk factors and the presence of dyspareunia or intermenstrual bleeding (Box 4).

Among the 95 presentations (54 women), there were 15 presentations (11 women) recorded by the practitioner as being a diagnosis of either PID alone (seven presentations, six women) or PID in conjunction an STI (two presentations, one woman) or urinary tract infection (six presentations, six women). The remainder were recorded as a urinary tract infection (25 presentations, 25 women), a sexually transmissible infection (12 presentations, 10 women), a condition unrelated to infection (20 presentations, 11 women) or had no diagnosis recorded (28 presentations, 11 women); in some of these presentations a dual diagnosis was recorded. Conversely, there were 20 presentations (19 women) out of the 224 that were diagnosed by the practitioner as PID, but had no documented evidence of PID other than lower abdominal pain (data not shown).

Of the 95 presentations, most (65) were managed by an RAN only, seven were referred to a GP, six were seen by a GP only, six by an Aboriginal health worker only, four by a sexual or women's health specialist only, and in seven presentations, the practitioner type was unknown. The rate of diagnosis did not differ by practitioner type (data not shown).

\section{Treatment for PID}

Correct treatment for PID, as specified in guidelines, was not administered in any of the 95 presentations (54 women) documented as having met the criteria for PID. Of the 35 presentations for which a PID diagnosis was recorded, none were documented to have received the recommended treatment (Box 2). In 12, correct medications were administered but either one was omitted or the wrong dose, duration or frequency of medication was prescribed; in 18, a single dose of azithromycin, amoxycillin and probenecid (the recommended syndromic treatment for gonorrhoea and chlamydia) was recorded; in four, pain relief only was recorded; and in one presentation, no treatment was recorded (data not shown)

\section{Discussion}

Our study is the most comprehensive assessment undertaken of PID diagnosis and management among Aboriginal women. In a community sample, we found that around one in 10 women in the age group at highest risk for PID presented to a primary health care centre with symptoms and investigation results consistent with PID at least once in a 2-year period. Most presentations were not investigated according to clinical guidelines for PID. As a result, cases likely to be PID were frequently missed and those that were detected were not treated according to guidelines. These episodes represent missed opportunities to prevent the long-term adverse reproductive outcomes associated with PID.

Our study is by no means the first to find that PID management presents a challenge in primary care.
An Australian study in an urban sexual health clinic found substantial variation among doctors in the number of PID diagnoses made compared with diagnoses of genital warts, ${ }^{12}$ a variation probably attributable to underdiagnosis of PID by some practitioners. A study in the United States reviewed emergency department records and found that only one-third of patients diagnosed with PID were prescribed antibiotics according to guidelines. ${ }^{13}$

Given the very high levels of PID indicated in our study and in earlier research from the $\mathrm{NT},{ }^{14,15}$ it is important to consider the reasons for the lack of guideline adherence observed in this study. Although provision of better or more accessible information has improved guideline adherence internationally ${ }^{16,17}$ it would probably not, on its own, change the situation markedly in central Australia, where PID-related education sessions have been widely available to both GPs and RANs for the past decade, as part of in-service training, and guidelines prompt clinicians to "think PID" in all relevant sections. ${ }^{10,18}$

A greater impact on PID management may arise through rethinking the guidelines to better reflect the clinical realities of remote primary health care. The current guidelines for diagnosis begin with the implication that a definitive diagnosis requires a bimanual pelvic examination, a procedure that many remote GPs and RANs may not be comfortable to readily perform, either because they do not have the training, or, if they do, they have limited recent experience. Furthermore, the individual clinical signs and symptoms stated in the guidelines lack sensitivity and specificity. ${ }^{19}$

A more pragmatic approach to PID diagnosis in this high-risk setting is based on the presence of lower abdominal pain, after the exclusion of other causes in sexually active women aged $14-34$ years. Additional information that would be of value includes younger age and background community STI prevalence, rather than specific signs and symptoms ${ }^{20}$ or individual sexual history. While this strategy would result in some women being unnecessarily treated for PID, it is consistent with the requirement for a high suspicion 
4 Proportion of presentations with recommended clinical assessments documented, presentations that met guideline criteria for diagnosing PID, and those with documented diagnoses of PID

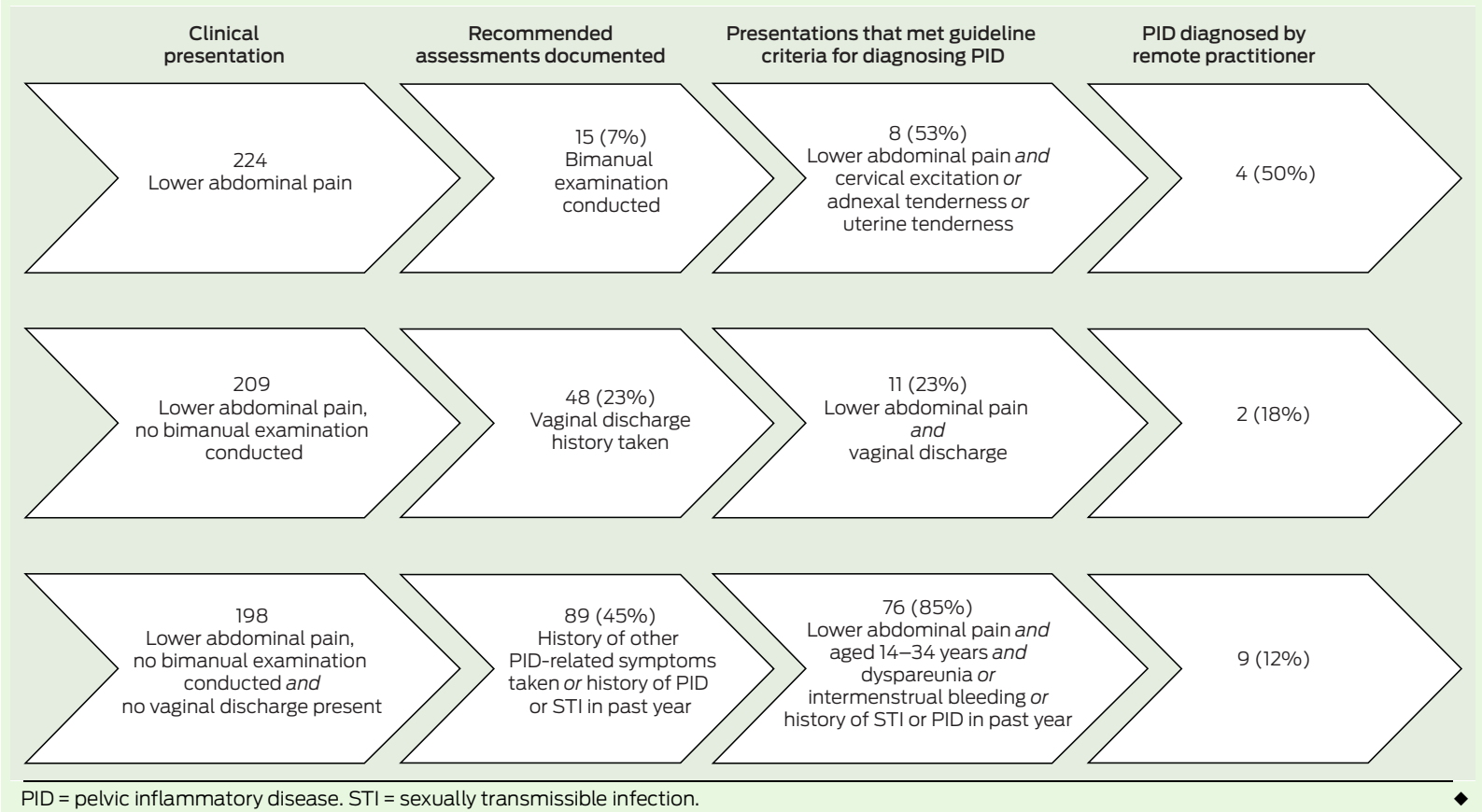

for detecting PID and a low threshold for diagnosis and treatment. It may also reduce the extent of delayed care which is associated with an increased risk of infertility and ectopic pregnancy. ${ }^{21}$

We acknowledge the broader challenges of working in remote primary care; the frequent staff turnover and multiple competing health priorities are well documented and have undoubtedly contributed to the gaps in PID management that we have identified. We suggest developing further clinical decision support in the form of pathways for the management of lower abdominal pain. In addition, the recent development of prompts and automated pathology and treatment pathways for PID within the patient management systems in the NT may also lead to improved management.

It is important to note that we did not review cases in which lower abdominal pain was not mentioned in the medical records. Some of these may have been additional missed opportunities for PID diagnosis. Furthermore, we assessed management as documented in the medical record, so did not record care that was provided but not documented. These methodological limitations mean that our findings should be interpreted with a degree of caution, even though they appear to be strong.

Ultimately, an improved ability to detect and manage PID in remote communities will reduce the high rate of hospitalisation for acute PID among Aboriginal women in the NT, and contribute to reducing serious reproductive sequelae. This change will require increased awareness, symptom recognition and revision of the current guidelines ${ }^{10}$ to reflect the reality of the remote situation. In addition, improving access to and delivery of a comprehensive sexual health program to help prevent bacterial STIs is imperative if we are to reduce the extent of PID. Until this is achieved, the reproductive health of Aboriginal women in central Australia will continue to be at risk.

Acknowledgements: We thank all communities and health services which participated in our study. John Kaldor is supported by an NHMRC research fellowship. Alice Rumbold is supported by the Jean B. Reid Fellowship from the University of Adelaide Medical Endowment Funds. Rebecca Guy is supported by an NHMRC postdoctoral fellowship. Our work was supported by the NHMRC (grant 568971). The views expressed are those of the authors and do not reflect the views of the NHMRC.

Competing interests: No relevant disclosures.

Received 9 Nov 2011, accepted 11 Jul 2012.

1 Paavonen J, Westrom L, Eschenbach D. Pelvic inflammatory disease. In: Holmes KK, Sparling PF, Stamm WE, et al, editors. Sexually transmitted diseases. 4th ed. New York: McGrawHill, 2008: 1017-1050.
2 Ness RB, Soper DE, Richter HE, et al. Chlamydia antibodies, chlamydia heat shock protein, and adverse sequelae after pelvic inflammatory disease: the PID Evaluation and Clinical Health (PEACH) Study. Sex Transm Dis 2008; 35: 129-135.

3 Simms I, Stephenson JM, Mallinson H, et al. Risk factors associated with pelvic inflammatory disease. Sex Transm Infect 2006; 82: 452-457.

4 Ness RB, Soper DE, Holley RL, et al. Effectiveness of inpatient and outpatient treatment strategies for women with pelvic inflammatory disease: results from the Pelvic Inflammatory Disease Evaluation and Clinical Health (PEACH) Randomized Trial. Am J Obstet Gynecol 2002 . 186: 929-937.

5 Sexual Health Society of Victoria. National management guidelines for sexually transmissible infections. 7th ed. Melbourne: Sexual Health Society of Victoria, 2008. http:// www.mshc.org.au/Portals/6/NMGFSTI.pdf (accessed Jul 2012).

6 Workowski KA, Berman S; Centers for Disease Control and Prevention. Sexually transmitted diseases treatment guidelines, 2010. MMWR Recomm Rep 2010; 59: 1-110.

7 Ross J, McCarthy G. UK National Guideline for the management of pelvic inflammatory disease. London: British Association for Sexual Health and HIV, 2011.

8 LiSQ, Gray NJ, Guthridge SL, Pircher SL. Avoidable hospitalisation in Aboriginal and non-Aboriginal people in the Northern Territory. Med J Aust 2009; 190: 532-536.

9 Guy R, Ward JS, Smith KS, et al. The impact of sexually transmissible infection programs in remote Aboriginal communities in Australia: a systematic review. Sex Health 2012; 9: 205-212.

10 Minymaku Kutju Tjukurpa Editorial Group. Minymaku kutju tjukurpa - women's business manual. 4th ed. Alice Springs: Congress Alukura Nganampa Health Council, 2008.

11 Centre for Disease Control. NT guidelines for the management of sexually transmitted infections 
in the primary health care setting. Darwin: Northern Territory Government, 2006.

12 Doxanakis A, Hayes RD, Chen MY, et al. Missing pelvic inflammatory disease? Substantial differences in the rate at which doctors diagnose PID. Sex Transm Infect 2008; 84: 518-523.

13 Kane BG, Degutis LC, Sayward HK, et al. Compliance with the Centers for Disease Control and Prevention recommendations for the diagnosis and treatment of sexually transmitted diseases. Acad Emerg Med 2004; 11: 371-377.

14 Kildea S, Bowden F. Reproductive health, infertility and sexually transmitted infections in indigenous women in a remote community in the Northern Territory. Aust N Z J Public Health 2000; 24: 382-386.

15 Mein J, Bowden FJ. A profile of inpatient STDrelated pelvic inflammatory disease in the Top End of the Northern Territory of Australia. Med J Aust 1997; 166: 464-467.

16 Trent M, Judy SL, Ellen JM, Walker A. Use of an institutional intervention to improve quality of care for adolescents treated in pediatric ambulatory settings for pelvic inflammatory disease. J Adolesc Health 2006; 39: 50-56.

17 Balamuth F, Zhao H, Mollen C. Toward improving the diagnosis and the treatment of adolescent pelvic inflammatory disease in emergency departments: results of a brief educational intervention. Pediatr Emerg Care 2010; 26: 85-92.
18 Central Australian Rural Practitioners Association. CARPA standard treatment manual: a clinic manual for primary health care practitioners in remote and rural communities in Central and Northern Australia. 4th ed. Alice Springs: CARPA, 2003.

19 Simms I, Warburton F, Weström L. Diagnosis of pelvic inflammatory disease: time for a rethink. Sex Transm Infect 2003; 79: 491-494.

20 Soper DE. Diagnosis and laparoscopic grading of acute salpingitis. Am J Obstet Gynecol 1991; 164: 1370-1376.

21 Hillis SD, Joesoef R, Marchbanks PA, et al. Delayed care of pelvic inflammatory disease is a risk factor for impaired infertility. Am J Obstet Gynecol 1993; 168: 1503-1509.

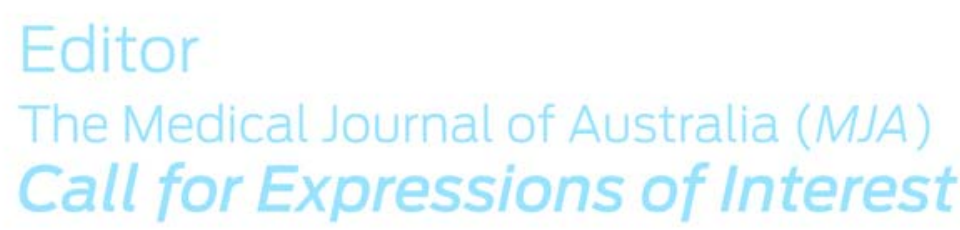

The Medical Journal of Australia is Australia's premier general medical journal.

It is the journal of the Australian Medical Association, which confers editorial independence, and is published by the Australasian Medical Publishing Company (AMPCo).

The AMPCo Board is seeking to appoint an outstanding individual to the position of

Editor of the Medical Journal of Australia on a full-time basis.

The Journal's offices are in the Sydney CBD.

Formal Qualifications:

- Formal medical qualifications essential

- Postgraduate medical qualifications desirable

Demonstrated Experience/Abilities:

- Experience in scientific investigation and research

- Experience in clinical medical practice

- Knowledge of the issues confronting the medical and health care community in Australia

- Highly developed English language and communication skills

-Substantial understanding of the requirements of academic publishing

- Strong leadership ability and experience managing a team of staff

- Proven time management, project management and people management skills

- Prior experience in medical publishing and editing highly desirable

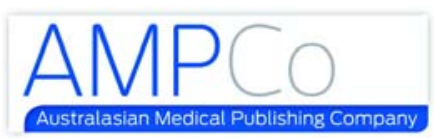

Please direct your expression of interest (by 9 February 2013) to:

Chairman, AMPCo Board

Tel: (02) 95626602 -mshepherd@ampco.com.au 